A metabolomic assessment of NAC154 transcription factor overexpression in field grown poplar stem wood

Judith Jervis, Sherry B. Hildreth, Xiaoyan Sheng, Eric P. Beers, Amy M. Brunner and Richard F. Helm*

Field grown transgenic and wild type poplar wood extracts were analyzed by LC-MS. NAC154 over-expression resulted in chemotypic and phenotypic changes, where arginine levels correlate with phenotype, suggesting NAC154 may be involved in dormancy/senescence processes.

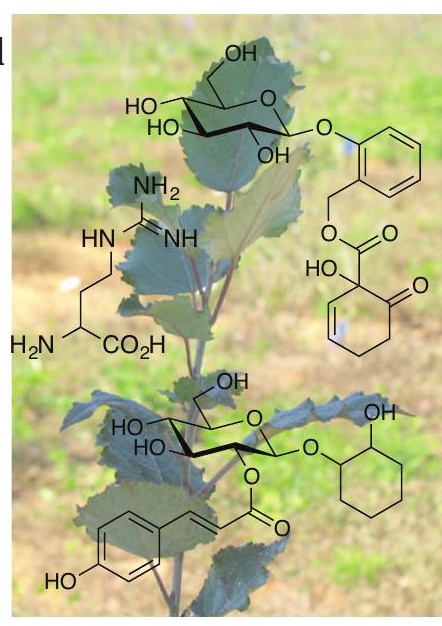

Control and NAC1540Es

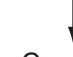

Current,

year growth

$\mathrm{MeOH}:$ water

$(1: 1, v / v)$

LC-MS

Mode

Mode

C18(+) 678

C18(-) 384

HILIC(+) 127

HILIC(-) 941 


\title{
A metabolomic assessment of NAC154 transcription factor overexpression in field grown poplar stem wood
}

\author{
Judith Jervis ${ }^{1}$, Sherry B. Hildreth ${ }^{2}$, Xiaoyan Sheng ${ }^{3}$, Eric P. Beers ${ }^{4}$, Amy M. Brunner ${ }^{3}$ and \\ Richard F. Helm ${ }^{1}$ \\ Departments of Biochemistry ${ }^{1}$, Biological Sciences ${ }^{2}$, Forest Resources and Environmental \\ Conservation $^{3}$ and Horticulture ${ }^{4}$, Virginia Tech, Blacksburg, Virginia 24061 USA \\ Corresponding Author: \\ Richard F. Helm \\ Department of Biochemistry \\ Life Sciences 1 \\ 970 Washington Street, SW \\ Blacksburg, VA 24061-0910 \\ E-mail: helmrf@vt.edu \\ Phone: 540-231-4088
}

FAX: 540-231-4043 


\begin{abstract}
Several xylem-associated regulatory genes have been identified that control processes associated with wood formation in poplar. Prominent among these are the NAC domain transcription factors (NACs). Here, the putative involvement of Populus NAC154, a co-ortholog of the Arabidopsis gene $S N D 2$, was evaluated as a regulator of "secondary" biosynthetic processes in stem internode tissues by interrogating aqueous methanolic extracts from control and transgenic trees. Comprehensive untargeted metabolite profiling was accomplished with a liquid chromatography-mass spectrometry platform that utilized two different chromatographic supports (HILIC and reversed phase) and both positive and negative ionization modes. Evaluation of current and previous year tissues provided datasets for assessing the effects of NAC154 overexpression in wood maturation processes. Phenolic glycoside levels as well as those of oligolignols, sucrose and arginine were modulated with phenotypic and chemotypic traits exhibiting similar trends. Specifically, increased levels of arginine in the NAC154 overexpressing tissues supports a role for the transcription factor in senescence/dormancyassociated processes.
\end{abstract}

Keywords: poplar; Populus tremula $x$ P. alba; Salicaceae; wood; arginine; LC-MS; metabolomics; NAC transcription factor

\title{
1. Introduction
}

The manipulation of both growth and maturation processes in woody tissues has the potential to increase biomass productivity as well as provide targeted wood and biomass end products (Costa et al., 2013; Van Acker et al., 2014; Wilkerson et al., 2014). Such research endeavors require tools that evaluate wood biochemical processes, which oftentimes necessitates the use of analytical methodologies that allow for assessment of metabolite pools. One technique that can provide such insights into woody tissue growth and maturation is liquid chromatography-mass spectrometry (Morreel et al., 2006; Robinson and Mansfield, 2011). Use of such technologies can identify features (or ions) that are significantly correlated with sample type. That said, feature and metabolite identification processes oftentimes have two problems, each with specific challenges. While it is relatively straightforward to identify ions that are different between treatments or genotypes, the identification process is complicated in poplar metabolomics by the lack of authentic standards and a species specific mass spectrometry database, as are available for plants such as rice, soybean, wheat and Arabidopsis (Matsuda et al., 2009).

NAC transcription factors (TFs) are plant-specific modulators of developmental processes. Their name is derived from the concatenation of three separate genes; NO APICAL MERISTEM (from petunia), ATAF1/ATAF2, and CUP-SHAPED COTYLEDON2 (CUC2); the latter two in Arabidopsis. As a class, NAC proteins possess a conserved $N$-terminal DNA binding domain with a $C$-terminus containing an activation domain (Aida et al., 1997; Duval et al., 2002; Ernst et al., 2004). A recent genomic analysis of Populus identified 163 NAC genes that clustered into 

TFs, those that positively and negatively regulate secondary cell wall synthesis (Grant et al., 2010). NAC154 (one of two Populus co-orthologs of SECONDARY WALL-ASSOCIATED NAC DOMAIN PROTEIN2, SND2; Potri.017G016700; UniProt ID: B9MYC7) expression was observed in both developing secondary xylem and phloem fibers, and overexpression of NAC154 resulted in greenhouse-grown trees exhibiting reduced height, more phloem-cambial tissue and less xylem when compared to control plants. The gene has been implicated in tension wood related processes (Andersson-Gunneras et al., 2006; Grant et al., 2010), and interestingly, conversion of NAC154 to a transcriptional repressor was recently reported to also provide a reduced growth phenotype (Wang et al., 2013). Research efforts with poplar NACs as well as the Arabidopsis co-ortholog SND2/ANAC073 suggest a hierarchical transcriptional framework where SND2 expression is controlled to some degree by SND1 (Zhong et al., 2010; Zhong et al., 2008; Zhong et al., 2011).

The absence of poplar metabolomic databases along with the lack of available information on the effect of NAC TF over-expression on field-grown trees (P. tremula $x$ P. alba clone 717-1B4) led us to evaluate the extractable metabolites present in stem internode tissues of field-grown trees produced by control and NAC154 overexpressing trees (N154OE) in the year of harvest (2012) as well as in the previous year (2011). The metabolomic analyses were combined with field evaluations to directly compare the chemotype with the observed phenotype. The datasets obtained are available at the MetaboLights (Haug et al., 2013) data repository (Accession: MTBLS119).

\section{Results and Discussion}

\subsection{Protocol Development}

A metabolic profiling experiment by LC-MS is essentially an in-depth analysis of pre-selected time windows. As such, there are several technical issues that must be considered prior to sample collection. In order to gain some insight into the processes associated with perennial tissues, stem internodes were analyzed from current year biosynthesis as well as tissues initially produced the previous year. To control for temporal changes inherent in woody tissues transferring metabolites in a time-dependent fashion, all samples were collected at the same time of day (mid-morning) in late August, presumably when most growth and cell elongation processes have ceased in wood forming tissue. To ensure consistency in developmental stages among the trees sampled, stem wood selection (internodes) was based upon the leaf plastochron index (LPI) (Larson and Isebrands, 1971), an assessment that relies upon morphological development rather than age (Fig. 1). Field-collected internodes were placed in dry ice and returned to the laboratory at which point they were freeze-dried to a constant weight. The samples were then ground to a fine powder and extracted with a mixture of methanol:water $(1: 1, \mathrm{v} / \mathrm{v})$. We chose 
not to separate the phloem and xylem as quantitatively separating these tissues with stems harvested in late summer is quite difficult.

A rapid yet informative assessment of the aqueous methanol extracts was next carried out. Short chromatographic separations (less than 10 minutes), utilizing UPLC separation technologies were coupled to a mass spectrometer with a rapid duty cycle and high mass accuracy (Waters SynaptG2-S HDMS). Separation methodologies relied upon gradient-based reversed-phase $\left(\mathrm{C}_{18}\right)$ and hydrophilic interaction (HILIC) chromatographies, with each biological sample analyzed in triplicate. Analyses were initiated by several injections of a "master mix" comprised of equal volumes of each sample type to equilibrate the system, followed by randomized injections to complete one LC-MS mode analysis. All individual runs in each analysis mode were comprised of two separate MS experiments, the main experiment being a parent ion scan $(0.185 \mathrm{sec}$ scan, $0.015 \mathrm{sec}$ delay) in the mass range of 50-1800. The second experiment was the lock mass scan of leucine-enkephalin ( $0.10 \mathrm{sec}$ scan, $0.10 \mathrm{sec}$ delay), which occurred once every $20 \mathrm{sec}$. These analyses were followed by a set of $\mathrm{MS}^{\mathrm{E}}$ analyses (data-independent high energy fragmentation) at several levels of energy to assess fragmentation patterns, which aid in compound identification. Once an ionization mode was completed, the mass spectrometer was switched from one ionization mode to the other, employing several blanks injections prior to the samples. A re-injection of a sample after the runs were completed indicated that there was no loss of sample integrity from the start to the completion of the LC-MS work. Once the analysis of all samples in one chromatography mode was completed, the UPLC system was switched to the other chromatography mode and the analyses in both ionization modes was repeated along with the $\mathrm{MS}^{\mathrm{E}}$ experiments.

\subsection{LC-MS Overview and Structural Assignments}

Representative chromatograms in base peak mode are shown in Fig. 2 with selected structures shown in Fig. 3. The methods of separation are orthogonal in nature as is indicated by the elution of tremulacin (4) near the end of the reversed-phase separation, and near the beginning of the HILIC separation. Sucrose (10) and arginine (11) eluted near the end of separation in HILIC mode whereas the compounds eluted at the solvent front in reversed-phase mode. It can also be seen that samples analyzed by HILIC fractionations and positive ion modes tend to have increased peak widths relative to reversed-phase and negative ion modes. Positive electrospray ionization (ESI) tended to favor $[\mathrm{M}+\mathrm{H}]^{+},[\mathrm{M}+\mathrm{Na}]^{+}$and associated cluster ions (for example $\left.[2 \mathrm{M}+\mathrm{Na}]^{+}\right)$whereas the negative mode ESI favored $[\mathrm{M}-\mathrm{H}]^{-}$and $[\mathrm{M}+\text { formate }]^{-}$and associated clusters. In-source decay was observed with many metabolites, especially in positive ion mode, and in general $[\mathrm{M}+\mathrm{Na}]^{+}$and $[\mathrm{M} \text {-formate }]^{-}$were resistant to fragmentation in the $\mathrm{MS}^{\mathrm{E}}$ (data independent acquisition/fragmentation) and targeted $\mathrm{MS}^{2}$ experiments. Efforts to minimize $[\mathrm{M}+\mathrm{Na}]^{+}$adduct formation by ion exchange were not successful. Salicortin (5) and tremulacin (4) have been reported as the major acetone-soluble phenolics in Populus tremuloides internodes 
(Clausen et al., 1989), and based upon the ion intensities found in the extracts, they are abundant molecules in the methanolic extracts of control and overexpressing internodes as well. All ions identified in this study and their corresponding peak areas can be found in the Supplemental Materials.

Most of the compounds identified in this work are based upon exact mass determinations and fragmentation patterns, with efforts focused on ions that differed in abundance between the control and N154OE lines. Use of both positive and negative ion modes provided a means to assign $[\mathrm{M}+\mathrm{H}]^{+}$and/or $[\mathrm{M}-\mathrm{H}]^{-}$in an unambiguous way, avoiding the assignment of the predominant ion as either $[\mathrm{M}+\mathrm{H}]^{+}$and/or $[\mathrm{M}-\mathrm{H}]^{-}$when it was an adduct ion. Fragmentation studies beyond the initial $\mathrm{MS}^{\mathrm{E}}$ studies were approached in a stepwise manner. When $[\mathrm{M}+\mathrm{H}]^{+}$ and/or $[\mathrm{M}-\mathrm{H}]^{-}$ions were readily apparent, they were submitted to fragmentation studies. When resistant adduct ions were encountered, manual searches for the weaker corresponding $[\mathrm{M}+\mathrm{H}]^{+}$ or $[\mathrm{M}-\mathrm{H}]^{-}$ion were carried out to determine if they were present in high enough levels to obtain fragmentation data. If not, the orthogonal chromatography mode was evaluated, relying upon the reproducible nature of UPLC-MS for locating the ion(s) of interest.

The assignments of some features required a shallower gradient in order to separate isobaric or "near-isobaric" metabolites. One interesting case was that of salicortin (5) and the grandidentatin/purpurein diastereomers (2) (Fig. 4). Salicortin can be envisioned as being formed via acylation of salicin (1) with the 1-hydroxy-6-oxocyclohex-2-enecarboxylic acid ( $\mathrm{HCH}$ group), whereas the grandidentatin/purpurein diastereomers can be derived from the $p$ coumaroylation of the $\beta$-glucopyranoside of cis-1,2-cyclohexandiol. The pathways for both $\mathrm{HCH}$ and 1,2-dicylohexanol biosynthesis have not been established. These two classes of phenolic glycosides differ in monoisotopic mass by $0.0364 \mathrm{Da}$, a result of being derived from different phenolic glycoside precursors (Babst et al., 2010; Boeckler et al., 2011). Selected mass scans for each of these compounds in the negative ion mode along with an extended solvent gradient (to better separate the metabolites) indicated that multiple isobaric peaks were present at each mass (Fig. 4). The multiple peaks could be derived either from the intact molecules as [M$\mathrm{H}]^{-}$, diasteroisomers, regioisomers differing sites of acylation, or from in-source decay of larger metabolites such as tremulacin (4). The $\mathrm{HCH}$ moiety of salicortin-type molecules could be detected in fragmentation studies (targeted $\mathrm{MS}^{2}$ or $\mathrm{MS}^{\mathrm{E}}$ ) by the presence of the 1-hydroxy-6oxocyclohex-2-enecarboxylate anion (calc. 155.0350, obsd. 155.0348) whereas the characteristic feature of the $p$-coumarate derivatives (cis or trans) was the presence of the dehydrated anion of p-coumaric acid (calc. 145.0295, obsd. 145.0295).

A review of the literature indicates that the stereochemistry about the 1-position of the salicortintype $\mathrm{HCH}$ group has not been established for poplar metabolites, although a related dimer from the fruit of Idesia polycarpa was reported to have an S-configuration (Yamakoshi et al., 2010). In the case of grandidentatin/purpurein (2), the structures were originally reported as 
diastereomers of 1,2-cis-cyclohexandiol (Pearl and Darling, 1962, 1970); thus the exact stereochemical assignments are not established. More recently, related metabolites have been isolated and characterized that place the p-coumaroyl ester on both the 4- (Si et al., 2009) and 6(Bae and Kim, 2009) positions of the carbohydrate, however there is no information available regarding the cyclohexanediol stereochemistry.

\subsection{Significant Features in the NAC154 Overexpressing and Control Internodes and Comparison to Field Phenotypes}

The raw data collected from the four sets of analyses (reversed-phase and HILIC in both positive and negative ion modes) were processed by MarkerLynx (Waters) software to identify ions that met selection criteria related to ion peak width and minimum thresholds over the mass range of 50-1800 amu (see Supplemental Methods). The application of the selection criteria resulted in deisotoped datasets ranging from 384 ions in the reversed-phase negative mode data to 1277 ions in the HILIC positive mode data (Fig. 1 and Supplemental Materials). Many of these ions corresponded to the same metabolite due to both adduct formation and in-source decay. In order to assess the overall data quality and experimental variability, Principal Components Analysis (PCA) of the HILIC data from the N154OE internodes for variation associated with field location and between internodes was performed (Fig. 4). While there was little correlation between field location and the N154OE transgenics, there was a clear separation of the transgenics based upon year of growth. These results suggest that the local conditions within the plot were the same for all trees, indicating this is not a key variable for further analysis. However, the internode growth year resulted in a clean PCA separation, indicating that comparing across the year of deposition (current vs. previous year wood) may provide insight into wood maturation processes.

The main ions/metabolites that were found to be different and detected as parent ions or adducts are shown in Table 1, arranged according to metabolite and LC-MS method. Three comparisons are shown, two compare the N154OE and wild type (WT) lines based upon initial year of growth, and the third compares current versus previous year wood regardless of genotype. In general, if an identified metabolite was found to be significantly different in both HILIC and reversed-phase analyses, they trended in the same direction, supporting the claim that the developed protocol is robust. A case in point is $p$-coumaroyl $\beta$-D-glucopyranoside (8), which eluted coincidentally at 2.81 minutes in both the HILIC and reversed-phase (RP) separations. This metabolite was present in higher levels in previous year growth tissue relative to current year wood when compared regardless of genotype.

When comparing the N154OE lines with the controls for current year growth, the metabolites identified were generally lower in abundance in the transgenic lines. The main exception to this trend was arginine (11), which was detected in 20-fold higher amounts in the HILIC-MS positive 
ion mode analyses (Table 1) (discussed below). Field phenotype and laboratory chemotype data are further compared in Fig. 5. Overexpression of NAC154 generated a set of transgenic trees with varying degrees of height and stem diameter reductions relative to the WT lines (Fig. 5A). The trees with NAC154 overexpressed also exhibited smaller composite leaf angles, meaning that adaxial leaf surfaces (top face of the leaf) were closer or more upright to the main stem relative to the controls. Chemotypic assessment of the same set of trees by PCA of the reversedphase separations in positive ion mode for the same transgenic trees are shown in Fig. 5B (for all ion modes and chromatographic separations see Supplemental Fig. S1). The older tissues (previous year wood, left panel), in particular, cluster in a way that mirrors the phenotypic data. The samples that have a greater height and stem diameter reduction are further from the control samples on the plot. The independent grouping of the samples in the older tissue PCA suggests the chemotypes and phenotypes correlate with one another.

The reduction in tree biomass due to NAC154 overexpression could have resulted from a decreased rate of growth throughout the growing season, early growth cessation, or a combination of these two. Seasonal leaf senescence and dormancy in poplar are correlated with increasing levels of arginine (11) in stem wood, probably serving as an $\mathrm{N}$ storage compound (Couturier et al., 2010). Thus the observed increase in arginine levels suggests that the reduced size of transgenic poplar is associated with some characteristics of dormancy in stems. As can be seen in Fig. 5A (right panel), the arginine levels correlate with phenotypic severity. In particular, composite leaf angle (CLA) showed the strongest inverse correlation with arginine peak area $(\mathrm{R}=-0.7937 ; \mathrm{p}=0.0012)$, and height and diameter were moderately inversely correlated with arginine level $(\mathrm{R}=-0.6 ; \mathrm{p}=0.04$; Supplemental Fig. S2). That NAC154 overexpression is linked with increased arginine (11) levels is a novel discovery made possible by the untargeted metabolite profiling. This finding provides insight regarding xylem-associated TFs as mediators of crosstalk between wood formation and overall plant growth and development, and highlights the importance of investigating dormancy control as a key to domestication of trees for enhanced woody biomass production (Ragauskas et al., 2006).

Populus species accumulate high levels of phenolic glycosides as a constitutive defense against herbivores and the levels of these compounds accumulating in bark show seasonal and genotypic variation (Boeckler et al., 2011; Keefover-Ring et al., 2014). The reduced levels observed in the $\mathrm{N} 154 \mathrm{OE}$ lines might also reflect an earlier cessation of growth relative to WT trees. While the aforementioned increase in arginine (11) levels and the reduced accumulation of phenolic glycosides point to the possibility of early growth cessation, we have not yet determined the basis of the connection between such chemotypic features, NAC154 overexpression, and reduced plant growth. In addition to direct effects of NAC154 overexpression, various abiotic stresses interacting with $N A C 154$ overexpression may have induced earlier growth cessation and changes in metabolites. For example, reduced photosynthetic carbon capture in the N154OE lines 
resulting from altered light interception by more vertically oriented leaves (Fig. 5A), could reduce growth and affect metabolites.

If a metabolite was found to be higher in abundance in WT wood in the current year samples, in general the trend remained in the older tissues. However, the total number of metabolites that were significantly different between the WT and N154OE transgenics was greater in the previous year wood, (13 vs. 9; Table 1) with most of the additional identified metabolites (e.g., pinoresinol hexoside, 12) found to be present in higher amounts in the N154OE tissues. Notably, in contrast to current year wood, arginine (11) levels did not differ for WT vs. N154OE transgenics in previous year wood (Table 1; compare WT:OE, Prev Yr to WT:OE, Curr Yr).

Differences in metabolite levels occurring between WT and N154OE lines were highlighted above. Overall, however, when the transgenic lines were compared to WT in order to determine the metabolites that changed in levels based upon growth years, relatively few metabolites were significantly different (data not shown). Considering this observation, it can be hypothesized that the features that differed in the samples were related more to growth year than NAC154 overexpression and consequently all genotypes were analyzed as a group against growth year. This analysis (Table 1, last column) provided multiple differences including higher levels of oligolignols in the older wood (Morreel et al., 2010; Morreel et al., 2004). The overall oligolignol profile obtained was similar to that reported previously for greenhouse-grown trees (Morreel et al., 2004) although the relative peak abundances were different. This could be due to actual ratio differences, the different methods of extraction and analysis, or a combination of the above. That oligolignols are more prevalent in previous year wood relative to current year wood suggests that lignification processes differ across growth years. It was also determined that sucrose (10) levels were higher in current year relative to previous year wood samples (Table 1), but were not significantly correlated with NAC154 over-expression. This may be a mass balance issue as there is more non-living tissues in previous year wood, which would presumably lack sucrose, making the levels lower in the older tissues. However, seasonal cessation of wood formation in poplar is associated with reduced sucrose levels (Deslauriers et al., 2009) and older wood may reach the cessation stage prior to new growth.

The metabolomics data permits the generation of several hypotheses concerning the connection between NAC154 function and the observed decreases in leaf angle and tree size. Leaf development in poplar and many eudicots is characterized by a gradual shift in orientation from upright leaf primordia toward horizontal or even pendulous orientation at maturity. Considering the observed increase in arginine content of the N154OE trees as a marker for earlier growth cessation, several aspects of growth and development, including attainment of normal orientation of mature leaves, may have been inhibited by early dormancy in the transgenic trees. Overexpression of transcriptional repressor chimeras SND2-SDRX in Arabidopsis (Zhong et al., 2008) and NAC154-SDRX in poplar (Wang et al., 2013) reportedly reduced xylem cell wall 
thickness. However, a complementary increase in xylem cell wall thickness in N154OE plants (Grant et al., 2010) was not observed, as would be predicted based on results from the dominant repression experiments. Thus it appears that NAC154 is not simply a positive regulator of xylem cell wall thickness. Moreover, the observations that both dominant repression and overexpression of NAC154 resulted in reduced plant size (Grant et al., 2010; Wang et al., 2013), and that downregulation of $\mathrm{NACl} 154$ is associated with tension wood production (AnderssonGunneras et al., 2006; Grant et al., 2010) raise additional questions about the role(s) of NAC154 and the possibility that the absolute level of expression and species-specific effects are important determinants of the phenotypes of NAC154 transgenic lines (Hussey et al., 2011). Additional loss- and gain-of-function experiments with NAC154 are necessary to more precisely determine the role of this xylem-associated NAC domain TF and the target pathways responsible for the metabolite profiles and growth-suppressive effects reported here.

\section{Conclusions}

The availability of the complete poplar genome sequence coupled with facile transgenesis has made poplar a useful model for understanding wood biochemical processes. As research moves from the laboratory to the field, baseline chemotypic information is required to assess the metabolic outputs of targeted gene manipulation as well as the effects of environmental variables on poplar growth and development (Keefover-Ring et al., 2014). As little information was available for internode wood metabolites via LC-MS approaches at the start of this investigation, it was instructive to obtain and make accessible a poplar metabolome database, and in doing so also evaluate the effects of the over-expression of a TF involved in wood maturation. The analysis of methanol:water extracts from whole internodes using a non-targeted approach was used to compare and contrast the effects of NAC154 overexpression in stems over a two year growth period. The use of UPLC technology coupled to a rapid-scanning high mass accuracy mass spectrometer allowed for the development of an exact mass-retention time map for poplar internode wood extracts. Statistical analysis of the predominant ions in the datasets indicated clear differences between growth years as well as with N154OE, with the data suggesting possible roles for NAC154 in growth cessation processes. There was a direct correlation between arginine (11) levels and field phenotype (height and composite leaf angle) clearly demonstrating that chemotypic evaluation of field grown poplar can be used in combination with phenotypic data to drive further understanding of wood biochemical processes. The dataset obtained (MetaboLights: MTBLS119) provides an initial resource for further annotation of the poplar metabolome as well as aiding in further studies on the effects of environmental conditions and/or genetic manipulation on poplar growth and development.

\section{Experimental}

\subsection{Phenotypic assessment of poplar transgenics}


P. tremula $\times$ P. alba clone 717-1B4 was the genotype used for all studies and the production of transgenics overexpressing Populus NAC154 (N154OE transgenics) as previously described (Grant et al., 2010). The transgenic events generated (i.e., independent insertion events) and non-transgenic WT control plants were propagated in tissue culture, acclimated to soil and grown in a glasshouse for two months before being planted at a field site in Critz, Virginia, USA, in May 2010. Ten ramets of WT trees and four ramets of each N154OE transgenic event were transplanted in two pairs, with each pair being placed randomly on the site at a $2.4 \times 2.4 \mathrm{~m}$ spacing. Additional types of transgenic poplars (i.e., with different transgenes) were included (data not shown) in this study for a total of 260 test trees surrounded by two border rows. Herbicide application and manual cultivation were used to control weeds. Tree heights were measured using a height pole, and stem diameter $(5 \mathrm{~cm}$ above ground level) using a caliper in October 2012. ImageJ was used to measure angles of petioles and blades of leaves using photographs taken in October 2011. For each leaf, two angles were measured: 1) between the adaxial side of the leaf petiole and the main stem, and 2) between the adaxial side of the leaf blade and a line extended from the petiole. The resulting values were summed for each leaf to yield a composite leaf angle (CLA). Thus the smaller the CLA value, the closer the adaxial leaf surface was to the main stem. Statistical analyses were done using JMP Pro 10 ((SAS Institute, Inc.). One-way analysis of variance (ANOVA) and unpaired t-test was used to determine the effect of the transgene (35Spro:NAC154). The response (Y-data) was the average of the two ramets in a pair, resulting in two independent data points for each transgenic event, and five data points for the control trees. To estimate and test differences between transgenic event and WT means, we used the LSMEANS protocol and Dunnett's adjustment.

\subsection{Poplar internode sample preparation}

Samples were collected from the field according to the leaf plastochron index (LPI), tagged and frozen in dry ice. For current year shoot segments, LPI 1 was defined as the first leaf below the shoot tip that was at least $1 \mathrm{~cm}$ in length (internode 1 would be directly above this), and counted down to collect internode 12. For the previous year growth, internode 1 was assigned as below the terminal bud scar, counting down the stem to internode 7 . Since trees showed different amounts of growth (number of internodes) in a given year, a continuous internode counting down from the shoot tip to the previous year's growth was not applicable. Samples in the laboratory were stored at $-80{ }^{\circ} \mathrm{C}$ prior to processing, which was initiated by freeze-drying to a constant weight. Twigs were flash frozen in $\mathrm{N}_{2}$ and subsequently ground in a P14 mill (Pulveristette 14; Fritsch) with a $0.5 \mathrm{~mm}$ sieve at maximum speed $(20,000 \mathrm{rpm})$ to generate a fine powder. The samples were collected in labeled, pre-weighed $1.5 \mathrm{~mL}$ centrifuge tubes, flash frozen in $\mathrm{N}_{2}$ and dried, after which the mass was recorded. Sample extraction was performed on $10 \mathrm{mg}$ fractions using (50:50 LCMS grade $\mathrm{MeOH}: \mathrm{H}_{2} \mathrm{O}, 1 \mathrm{~mL}$ ). The tube contents were mixed by vortexing, placed in a shaker (Boekel Sci; Feasterville, PA) and incubated 200 minutes at 30 ${ }^{\circ} \mathrm{C}$. The tubes were then removed, vortexed briefly and allowed to sit overnight at room 
temperature. The samples were then vortexed and centrifuged $(13,000 \mathrm{x} \mathrm{g}, 5 \mathrm{~min}, \mathrm{RT})$ with the extracts $(450 \mu \mathrm{L})$ transferred to prelabeled, ethanol-washed tubes. The samples were then concentrated via centrifugal concentration until approximately $200 \mu \mathrm{L}$ remained and were then flash frozen in $\mathrm{N}_{2}$ and dried overnight on high vacuum line with a $\mathrm{N}_{2}$ trap. The resulting dried extract was then weighed to determined yield.

\subsection{Reversed-phase Separations}

The extract was reconstituted with $50 \mathrm{nM}$ acetaminophen (internal standard) in $0.1 \%$ aq. $\mathrm{HCO}_{2} \mathrm{H}: \mathrm{CH}_{3} \mathrm{CN}(9: 1, \mathrm{v} / \mathrm{v})$ with the volume based upon the weight of the dried extract (100 $\mu \mathrm{L} / \mathrm{mg}$ dry extract). Dissolution was aided by vortexing followed by sonication (10 minutes). The samples were then centrifuged $(13,000 \mathrm{x}$ g; $10 \mathrm{~min}$; RT) and an aliquot was removed (100 $\mu \mathrm{L})$ making sure not to disturb any precipitate. The sample was further diluted (10x) with $50 \mathrm{nM}$ acetaminophen in $0.1 \% \mathrm{HCO}_{2} \mathrm{H}: \mathrm{CH}_{3} \mathrm{CN}(9: 1, \mathrm{v} / \mathrm{v})$ and transferred to a LC-MS grade autosampler vial. A "MasterMix" was constructed from a single representative sample from each class. This sample is used to equilibrate the LC-MS system and is also used for statistical validation.

Metabolite separations were performed with an UPLC (Waters I-Class) equipped with a BEH $\mathrm{C} 18$ column $(1.7 \mu \mathrm{m} ; 2.1 \mathrm{~mm} \times 50 \mathrm{~mm}$; Waters $)$. The mobile phases were $\mathrm{H}_{2} \mathrm{O}$ with $0.1 \%$ $\mathrm{HCO}_{2} \mathrm{H}$ (Solvent A) and $\mathrm{CH}_{3} \mathrm{CN}$ (Solvent B) at $200 \mathrm{uL} / \mathrm{min}$. The separation initially started with 95\% A (0-1 min) with a linear ramp to $85 \% \mathrm{~A}(1.0-1.5 \mathrm{~min})$, followed by a second ramp to $40 \% \mathrm{~A}$ (1.5-6.9 $\mathrm{min})$. The gradient was rapidly ramped to $10 \% \mathrm{~A}(6.9-7.0 \mathrm{~min})$ where it was held for $0.5 \mathrm{~min}(7.0-7.5 \mathrm{~min})$. Re-equilibration involved ramping back to $95 \% \mathrm{~A}(7.5-8.0 \mathrm{~min})$ and holding at $95 \% \mathrm{~A}$ for $2 \mathrm{~min}(8.0-10.0 \mathrm{~min})$. Injection volumes were $1 \mathrm{uL}$, the column temperature was $40{ }^{\circ} \mathrm{C}$, with the autosampler maintained at $8^{\circ} \mathrm{C}$. Under these conditions acetaminophen elutes at $2.07 \mathrm{~min}(\mathrm{~m} / \mathrm{z}=152.0708$ in positive mode and $\mathrm{m} / \mathrm{z}=150.0556$ in negative mode). Leucine-enkephalin was used as lock mass, scanning for $0.1 \mathrm{sec}$ every $20 \mathrm{sec}$. Extended run conditions (Fig. 3) were as follows: $95 \%$ A (0-1 min) with a linear ramp to $50 \% \mathrm{~A}$ (1.0-20 $\mathrm{min})$, followed by a second ramp to $1 \% \mathrm{~A}(20-25 \mathrm{~min})$ and a one minute hold. The gradient was then ramped to $95 \% \mathrm{~A}$ in one minute where it was held for $3 \mathrm{~min}$ (for reequilibration).

\subsection{HILIC Separations}

The extract was reconstituted with $0.1 \% \mathrm{HCO}_{2} \mathrm{H}: \mathrm{CH}_{3} \mathrm{CN}(1: 1, \mathrm{v} / \mathrm{v})$ with the volume based upon the weight of the dried extract $(100 \mu \mathrm{L} / \mathrm{mg}$ dry extract). Dissolution was aided by vortexing followed by sonication (10 minutes). The samples were then centrifuged (13,000 x g; $10 \mathrm{~min}$; RT) and an aliquot $(100 \mu \mathrm{L})$ was removed making sure not to disturb any precipitate. The sample was further diluted (10x) with $0.1 \% \mathrm{HCO}_{2} \mathrm{H}: \mathrm{CH}_{3} \mathrm{CN}(1: 1, \mathrm{v} / \mathrm{v})$ and transferred to a LCMS grade autosampler vial with an insert. A "MasterMix" was constructed from a single representative sample from each class. This sample is used to equilibrate the LC-MS system and is also used for statistical validation. 
Metabolite separations were performed with an UPLC (Waters I-Class) equipped with a BEH Amide $\left(1.7 \mu \mathrm{m}, 2.1 \mathrm{~mm} \times 50 \mathrm{~mm}\right.$; Waters). The mobile phases were water with $0.1 \% \mathrm{HCO}_{2} \mathrm{H}$ (Solvent A) and $\mathrm{CH}_{3} \mathrm{CN}$ with $0.1 \% \mathrm{HCO}_{2} \mathrm{H}$ (Solvent B) at $400 \mathrm{uL} / \mathrm{min}$. The separation initially started with $99 \% \mathrm{~B}(0-0.5 \mathrm{~min})$ with a linear ramp to $50 \% \mathrm{~B}(0.5-7.0 \mathrm{~min})$. The gradient was rapidly ramped to $99 \% \mathrm{~B}(7.0-7.1 \mathrm{~min})$ where it was held for $3.9 \mathrm{~min}$ (7.1-10 min). Injection volumes were $1 \mathrm{uL}$, the column temperature was $45^{\circ} \mathrm{C}$, and the autosampler maintained at $8^{\circ} \mathrm{C}$. Leucine-enkephalin was used as lock mass, scanning for $0.1 \mathrm{sec}$ every $20 \mathrm{sec}$.

\subsection{Mass Spectrometry}

A Waters SynaptG2-S HDMS instrument was used in high-resolution mode employing the extended dynamic range feature and electrospray ionization. Capillary voltages were of $2.4 \mathrm{kV}$ and $3.0 \mathrm{kV}$ for negative- and positive-ion modes, respectively, with a cone voltage of $40 \mathrm{~V}$. Cone and desolvation gas flows were 50 and $500 \mathrm{~L} / \mathrm{hr}$, respectively with source and desolvation gas temperatures at $125^{\circ} \mathrm{C}$ and $350^{\circ} \mathrm{C}$, respectively. The instrument was calibrated with sodium formate from 50 to $1800 \mathrm{~m} / z$. Infusion flow rate for lock mass was $5 \mu \mathrm{L} / \mathrm{min}$. Leucine enkephalin $(2 \mathrm{ng} / \mathrm{mL})$ is used as lock mass for positive $(\mathrm{m} / \mathrm{z} 556.2771)$ and negative $(\mathrm{m} / \mathrm{z}$ 554.2615 ) mode experiments with parameters set to $0.2 \mathrm{sec} / 20 \mathrm{sec}$ intervals. MS spectra are acquired in centroid mode from 50 to $1800 \mathrm{~m} / \mathrm{z}$ using a scan time of $0.2 \mathrm{~s}$.

\subsection{Ion Feature Selection, Data Processing}

The commercial MarkerLynx, MetaboLynx and QuanLynx software (Waters) were used to analyze the datasets obtained. If an authentic standard was available it was analyzed under the same conditions as described above. Ions that were identified as significantly different by the processing software were first confirmed manually and subsequently submitted to fragmentation analyses. See the Supplemental for details.

\subsection{Compound Identification}

Standards were used when available for exact mass-retention time confirmation. These included arginine (11), salicin (1), sucrose (10), tremulacin, $p$-coumaryl $\beta$-D-glucopyranoside $(\mathbf{8}$; courtesy of J. Ralph, Wisconsin). Master mix samples were also submitted the fragmentation studies using either targeted $\mathrm{MS}^{2}$ or data independent fragmentation $\left(\mathrm{MS}^{\mathrm{E}}\right)$ studies.

\section{Acknowledgements}

This work was supported by the Office of Science (BER), US Department of Energy, Grant No. DE-FG02-07ER64449 (to EPB, AMB and RFH). The mass spectrometry resources used in this work are maintained in part through funding by the Fralin Life Science Institute, the Agricultural Experiment Station Hatch Program, and the McIntire-Stennis Program at Virginia Tech. We thank Kyle Peer, Deborah Bird, and Clay Sawyers for preparation, maintenance and growth measurement of the field study, and Nina Wilson for measurements of composite leaf angles. 


\section{References}

Aida, M., Ishida, T., Fukaki, H., Fujisawa, H., Tasaka, M., 1997. Genes involved in organ separation in Arabidopsis: an analysis of the cup-shaped cotyledon mutant. Plant Cell 9, 841857.

Andersson-Gunneras, S., Mellerowicz, E. J., Love, J., Segerman, B., Ohmiya, Y., Coutinho, P. M., Nilsson, P., Henrissat, B., Moritz, T., Sundberg, B., 2006. Biosynthesis of cellulose-enriched tension wood in Populus: global analysis of transcripts and metabolites identifies biochemical and developmental regulators in secondary wall biosynthesis. Plant J 45, 144-165.

Babst, B. A., Harding, S. A., Tsai, C. J., 2010. Biosynthesis of phenolic glycosides from phenylpropanoid and benzenoid precursors in populus. J Chem Ecol 36, 286-297.

Bae, Y.-S., Kim, J.-K., 2009. Grandidentatin isomer from bark of suwon poplar (Populus alba L. x Populus glandulosa Uyeki). Holzforschung 63, 315-318.

Boeckler, G. A., Gershenzon, J., Unsicker, S. B., 2011. Phenolic glycosides of the Salicaceae and their role as anti-herbivore defenses. Phytochemistry 72, 1497-1509.

Clausen, T. P., Evans, T. P., Reichardt, P. B., 1989. A Simple Method for the Isolation of Salicortin, Tremulacin, and Tremuloiden from Quaking Aspen (Populus tremuloides). J Nat Prod 52, 207-209.

Costa, M. A., Marques, J. V., Dalisay, D. S., Herman, B., Bedgar, D. L., Davin, L. B., Lewis, N. G., 2013. Transgenic hybrid poplar for sustainable and scalable production of the commodity/specialty chemical, 2-phenylethanol. PLoS One 8, e83169.

Couturier, J., Doidy, J., Guinet, F., Wipf, D., Blaudez, D., Chalot, M., 2010. Glutamine, arginine and the amino acid transporter Pt-CAT11 play important roles during senescence in poplar. Ann Bot 105, 1159-1169.

Deslauriers, A., Giovannelli, A., Rossi, S., Castro, G., Fragnelli, G., Traversi, L., 2009. Intraannual cambial activity and carbon availability in stem of poplar. Tree Physiol 29, 1223-1235.

Duval, M., Hsieh, T. F., Kim, S. Y., Thomas, T. L., 2002. Molecular characterization of AtNAM: a member of the Arabidopsis NAC domain superfamily. Plant Molec. Biol. 50, 237 248.

Ernst, H. A., Olsen, A. N., Larsen, S., Lo Leggio, L., 2004. Structure of the conserved domain of ANAC, a member of the NAC family of transcription factors. EMBO Rep 5, 297-303.

Grant, E. H., Fujino, T., Beers, E. P., Brunner, A. M., 2010. Characterization of NAC domain transcription factors implicated in control of vascular cell differentiation in Arabidopsis and Populus. Planta 232, 337-352.

Hu, R., Qi, G., Kong, Y., Kong, D., Gao, Q., Zhou, G., 2010. Comprehensive analysis of NAC domain transcription factor gene family in Populus trichocarpa. BMC Plant Biol. 10, 145. 

2011. SND2, a NAC transcription factor gene, regulates genes involved in secondary cell wall development in Arabidopsis fibres and increases fibre cell area in Eucalyptus. BMC Plant Biol $11,173$.

Keefover-Ring, K., Ahnlund, M., Abreu, I. N., Jansson, S., Moritz, T., Albrectsen, B. R., 2014. No evidence of geographical structure of salicinoid chemotypes within Populus tremula. PLoS One 9, e107189.

Larson, P. R., Isebrands, J. G., 1971. The plastochron index as applied to developmental studies of cottonwood. Can J For Res 1, 1-11.

Matsuda, F., Yonekura-Sakakibara, K., Niida, R., Kuromori, T., Shinozaki, K., Saito, K., 2009. MS/MS spectral tag-based annotation of non-targeted profile of plant secondary metabolites. Plant J 57, 555-577.

Morreel, K., Goeminne, G., Storme, V., Sterck, L., Ralph, J., Coppieters, W., Breyne, P., Steenackers, M., Georges, M., Messens, E., Boerjan, W., 2006. Genetical metabolomics of flavonoid biosynthesis in Populus: a case study. Plant J. 47, 224-237.

Morreel, K., Kim, H., Lu, F., Dima, O., Akiyama, T., Vanholme, R., Niculaes, C., Goeminne, G., Inze, D., Messens, E., Ralph, J., Boerjan, W., 2010. Mass spectrometry-based fragmentation as an identification tool in lignomics. Anal Chem 82, 8095-8105.

Morreel, K., Ralph, J., Kim, H., Lu, F., Goeminne, G., Ralph, S., Messens, E., Boerjan, W., 2004. Profiling of oligolignols reveals monolignol coupling conditions in lignifying poplar xylem. Plant Physiol 136, 3537-3549.

Pearl, I. A., Darling, S. F., 1962. Studies on the barks of the family Salicaceae. V. Grandidentatin, a new glucoside from the bark of Populus grandidentata. J. Org. Chem. 27, 1806-1809.

Pearl, I. A., Darling, S. F., 1970. Purpurein, a new glucoside from the bark of Salix purpurea. Phytochemistry 9, 853-856.

Ragauskas, A. J., Williams, C. K., Davison, B. H., Britovsek, G., Cairney, J., Eckert, C. A., Frederick, W. J., Jr., Hallett, J. P., Leak, D. J., Liotta, C. L., Mielenz, J. R., Murphy, R., Templer, R., Tschaplinski, T., 2006. The path forward for biofuels and biomaterials. Science 311, 484489.

Robinson, A. R., Mansfield, S. D., 2011. Metabolomics in Poplar. In: Joshi, C. P., DiFazio, S. P., Kole, C. (Eds.), Genetics, Genomics and Breeding of Poplar. CRC Press, pp. 166-191.

Si, C. L., Wu, L., Zhu, Z. Y., 2009. Phenolic glycosides from Populus davidiana bark. Biochem Syst Ecol 37, 221-224.

Van Acker, R., Leple, J. C., Aerts, D., Storme, V., Goeminne, G., Ivens, B., Legee, F., Lapierre, C., Piens, K., Van Montagu, M. C., Santoro, N., Foster, C. E., Ralph, J., Soetaert, W., Pilate, G., 
Boerjan, W., 2014. Improved saccharification and ethanol yield from field-grown transgenic poplar deficient in cinnamoyl-CoA reductase. Proc Natl Acad Sci U S A 111, 845-850.

Wang, H. H., Tang, R. J., Liu, H., Chen, H. Y., Liu, J. Y., Jiang, X. N., Zhang, H. X., 2013. Chimeric repressor of PtSND2 severely affects wood formation in transgenic Populus. Tree Physiol 33, 878-886.

Wilkerson, C. G., Mansfield, S. D., Lu, F., Withers, S., Park, J. Y., Karlen, S. D., GonzalesVigil, E., Padmakshan, D., Unda, F., Rencoret, J., Ralph, J., 2014. Monolignol ferulate transferase introduces chemically labile linkages into the lignin backbone. Science 344, 90-93.

Yamakoshi, H., Shibuya, M., Tomizawa, M., Osada, Y., Kanoh, N., Iwabuchi, Y., 2010. Total synthesis and determination of the absolute configuration of (-)-idesolide. Organic Letters 12, 980-983.

Zhong, R., Lee, C., Ye, Z. H., 2010. Functional characterization of poplar wood-associated NAC domain transcription factors. Plant Physiol 152, 1044-1055.

Zhong, R., Lee, C., Zhou, J., McCarthy, R. L., Ye, Z. H., 2008. A battery of transcription factors involved in the regulation of secondary cell wall biosynthesis in Arabidopsis. Plant Cell 20, 2763-2782.

Zhong, R., McCarthy, R. L., Lee, C., Ye, Z. H., 2011. Dissection of the transcriptional program regulating secondary wall biosynthesis during wood formation in poplar. Plant Physiol 157, 1452-1468. 


\section{Figure Captions}

Fig. 1. Overview of the Sampling and LC-MS Methodologies. Internodes were collected according to the leaf plastochron index (LPI). For the current year shoot segment, LPI 1 was the first leaf below the shoot tip that was at least $1 \mathrm{~cm}$ in length. Internode 1 was directly above this leaf, and counting down provided internode 12. For the previous year growth, internode 1 was directly below the terminal bud scar, and counting down the stem provided internode 7. Data processing provided between 384-1277 individual features (ions), depending on the analysis mode.

Fig. 2. LC-MS Separations in Reversed-Phase and HILIC Modes. The example chromatograms are presented in base peak ion mode. The two separation methods are orthogonal in nature as seen by the elution times of tremulacin (4). In the reversed-phase separations, sucrose and arginine eluted at the solvent front.

Fig. 3. Structures of Compounds.

Fig. 4. Salicortin and Grandidentatin/Purpurein Metabolites. The structures of grandidentatin/purpurein (2) and salicortin (5) are shown in Panel A with key diagnostic fragmentation ions. Panel B shows the full scan base peak ion chromatogram (i) along with selected ion traces for the intact metabolites (ii, iv) and the key fragment ions (iii, v), all utilizing a reversed-phase separation and negative ion mode. The extended gradient conditions used are described in the Experimental.

Fig. 5. Principal Components Analyses of the NAC154 Overexpressing Transgenic Lines Based Upon HILIC Separations. A, Negative ion mode analyses based upon field location (top) and year of growth (bottom). B, Positive ion mode analyses based upon field location (top) and year of growth (bottom). Samples segregate based upon growth year but not field location.

Fig. 6. Phenotypic and Chemotypic Assessments of the NAC154 Overexpressing Transgenic Trees. A, Characterization based upon tree height, stem diameter, composite leaf angle (CLA) and arginine peak areas. B, Principal components analyses of the LC-MS data (reversed phase, positive ion mode) for previous and current year samples. 
Table 1. Metabolites Identified as Changing in Relative Levels Due to NAC154 Over-Expression and Growth Year.

\begin{tabular}{|c|c|c|c|c|c|c|c|c|}
\hline Compound & RT & Mass & Mode & Ion & $\begin{array}{c}\text { ID }^{1} \\
\text { Level }\end{array}$ & $\begin{array}{l}\text { WT:OE } \\
\text { Current }^{2}\end{array}$ & $\begin{array}{c}\text { WT:OE } \\
\text { Previous }\end{array}$ & $\begin{array}{c}\text { Curr:Prev } \\
\text { All genotypes }\end{array}$ \\
\hline arginine (11) & 5.84 & 175.1194 & HILIC-(+) & {$[\mathrm{M}+\mathrm{H}]$} & 1 & 0.05 & --- & --- \\
\hline cinnamoyl hexoside (9) & 3.82 & 333.0945 & $\mathrm{RP}-(+)$ & {$[\mathrm{M}+\mathrm{Na}]$} & 2 & --- & --- & 0.29 \\
\hline \multirow[t]{3}{*}{$p$-coumaroyl $\beta$-D-Glc $p$ (8) } & 2.81 & 325.0920 & HILIC-(-) & {$[\mathrm{M}-\mathrm{H}]$} & 1 & 2.8 & 1.9 & 0.32 \\
\hline & 2.81 & 325.0921 & RP-(-) & {$[\mathrm{M}-\mathrm{H}]$} & 1 & --- & --- & 0.49 \\
\hline & 2.82 & 349.0896 & HILIC-(+) & {$[\mathrm{M}+\mathrm{Na}]$} & 1 & --- & --- & 0.37 \\
\hline \multirow[t]{2}{*}{ grandidentatin/purpurein (2) } & 2.43 & 423.1650 & HILIC-(-) & {$[\mathrm{M}-\mathrm{H}]$} & 2 & 2.2 & 1.4 & --- \\
\hline & 2.62 & 447.1607 & HILIC-(+) & {$[\mathrm{M}+\mathrm{Na}]$} & 2 & --- & 0.8 & --- \\
\hline populoside (15) & 2.85 & 447.1287 & HILIC-(-) & {$[\mathrm{M}-\mathrm{H}]$} & 2 & --- & --- & 0.53 \\
\hline \multirow[t]{2}{*}{ populoside B/trichocarposide $(\mathbf{1 7 , 1 8})$} & 4.60 & 455.1311 & $\mathrm{RP}-(+)$ & {$[\mathrm{M}+\mathrm{Na}]$} & 2 & --- & --- & 0.50 \\
\hline & 4.64 & 431.1339 & RP-(-) & {$[\mathrm{M}-\mathrm{H}]$} & 2 & --- & --- & 0.48 \\
\hline pinoresinol hexoside (12) & 3.75 & 519.1862 & RP-(-) & {$[\mathrm{M}-\mathrm{H}]$} & 2 & --- & 0.83 & --- \\
\hline sakuranin/neosakuranin $(\mathbf{1 3 , 1 4})$ & 2.58 & 447.1287 & HILIC-(-) & {$[\mathrm{M}-\mathrm{H}]$} & 2 & 2.1 & --- & --- \\
\hline \multirow[t]{2}{*}{ salicin (1) } & 2.50 & 309.0947 & $\mathrm{RP}-(+)$ & {$[\mathrm{M}+\mathrm{Na}]$} & 1 & --- & --- & 2.0 \\
\hline & 2.90 & 309.0949 & HILIC-(+) & {$[\mathrm{M}+\mathrm{Na}]$} & 1 & --- & --- & 2.4 \\
\hline salicortin (5) & 3.57 & 447.1260 & $\mathrm{RP}-(+)$ & {$[\mathrm{M}+\mathrm{Na}]$} & 2 & 1.2 & --- & --- \\
\hline \multirow[t]{2}{*}{ sucrose (10) } & 5.04 & 341.1080 & HILIC-(-) & {$[\mathrm{M}-\mathrm{H}]$} & 1 & --- & --- & 1.5 \\
\hline & 5.05 & 365.1058 & HILIC-(+) & {$[\mathrm{M}+\mathrm{Na}]$} & 1 & --- & --- & 1.3 \\
\hline \multirow[t]{3}{*}{ tremulacin $(\mathbf{4})$} & 1.85 & 573.1605 & HILIC-(-) & [M+formate $]$ & 1 & --- & --- & 2.3 \\
\hline & 5.34 & 551.1525 & $\mathrm{RP}-(+)$ & {$[\mathrm{M}+\mathrm{Na}]$} & 1 & --- & 1.2 & --- \\
\hline & 5.38 & 527.1549 & RP-(-) & {$[\mathrm{M}-\mathrm{H}]$} & 1 & 1.3 & 1.5 & 1.9 \\
\hline lignan/neolignan & 2.87 & 431.1309 & $\mathrm{RP}-(+)$ & {$[\mathrm{M}+\mathrm{Na}]$} & 2 & --- & --- & 0.37 \\
\hline tetrameric oligolignol & 5.19 & 833.2983 & $\mathrm{RP}-(+)$ & {$[\mathrm{M}+\mathrm{Na}]$} & 2 & --- & --- & 0.48 \\
\hline trimeric oligolignol & 4.96 & 605.1995 & $\mathrm{RP}-(+)$ & {$[\mathrm{M}+\mathrm{Na}]$} & 2 & --- & --- & 0.40 \\
\hline sakuranin/neosakuranin (fragment) & 2.60 & 287.0918 & HILIC-(+) & {$[\mathrm{M}+\mathrm{H}]$} & 2 & 3.0 & 2.1 & --- \\
\hline syringic acid hexoside & 2.43 & 359.0974 & RP-(-) & {$[\mathrm{M}-\mathrm{H}]$} & 2 & --- & 0.71 & --- \\
\hline Unknown (feruloylated salicin) & 4.70 & 485.1415 & $\mathrm{RP}-(+)$ & {$[\mathrm{M}+\mathrm{Na}]$} & 3 & --- & --- & 0.36 \\
\hline Unknown (flavonone disaccharide) & 2.46 & 617.1841 & HILIC-(+) & {$[\mathrm{M}+\mathrm{Na}]$} & 3 & --- & 2.4 & 0.36 \\
\hline Unknown (flavonone) & 3.97 & 287.0917 & $\mathrm{RP}-(+)$ & $?$ & 4 & 3.4 & 0.40 & --- \\
\hline Unknown (p-coumaroyl ester) & 3.93 & 421.1493 & RP-(-) & {$[\mathrm{M}-\mathrm{H}]$} & 4 & 0.60 & --- & --- \\
\hline Unknown (benzoyl ester) & 4.01 & 429.1155 & $\mathrm{RP}-(+)$ & {$[\mathrm{M}+\mathrm{Na}]$} & 4 & --- & 1.2 & --- \\
\hline Unknown (apocarotenoid?) & 3.05 & 431.1912 & RP-(-) & {$[\mathrm{M}+$ formate $]$} & 4 & --- & 0.67 & --- \\
\hline Unknown (lusitanicoside-like) & 3.74 & 465.1728 & $\mathrm{RP}-(+)$ & {$[\mathrm{M}+\mathrm{Na}]$} & 4 & --- & --- & 0.45 \\
\hline Unknown (lusitanicoside-like) & 3.78 & 487.1800 & RP-(-) & [M+formate $]$ & 4 & --- & --- & 0.48 \\
\hline Unknown (apocarotenoid?) & 3.75 & 575.2336 & RP-(-) & {$[\mathrm{M}-\mathrm{H}]$} & 4 & --- & 0.71 & --- \\
\hline \multirow[t]{2}{*}{ Unknown } & 5.21 & 591.2077 & RP-(-) & [M+formate $]$ & 4 & --- & --- & 0.59 \\
\hline & & & & Total ions & & 9 & 13 & 21 \\
\hline
\end{tabular}

${ }^{1}$ Based on Sumner et al. (2007): 1. Identified; 2, Putatively annotated; 3, Putatively characterized; 4. Unknown compounds. ${ }^{2}$ Factors of change with p-values $<0.01$ as calculated by Student's t-test. 


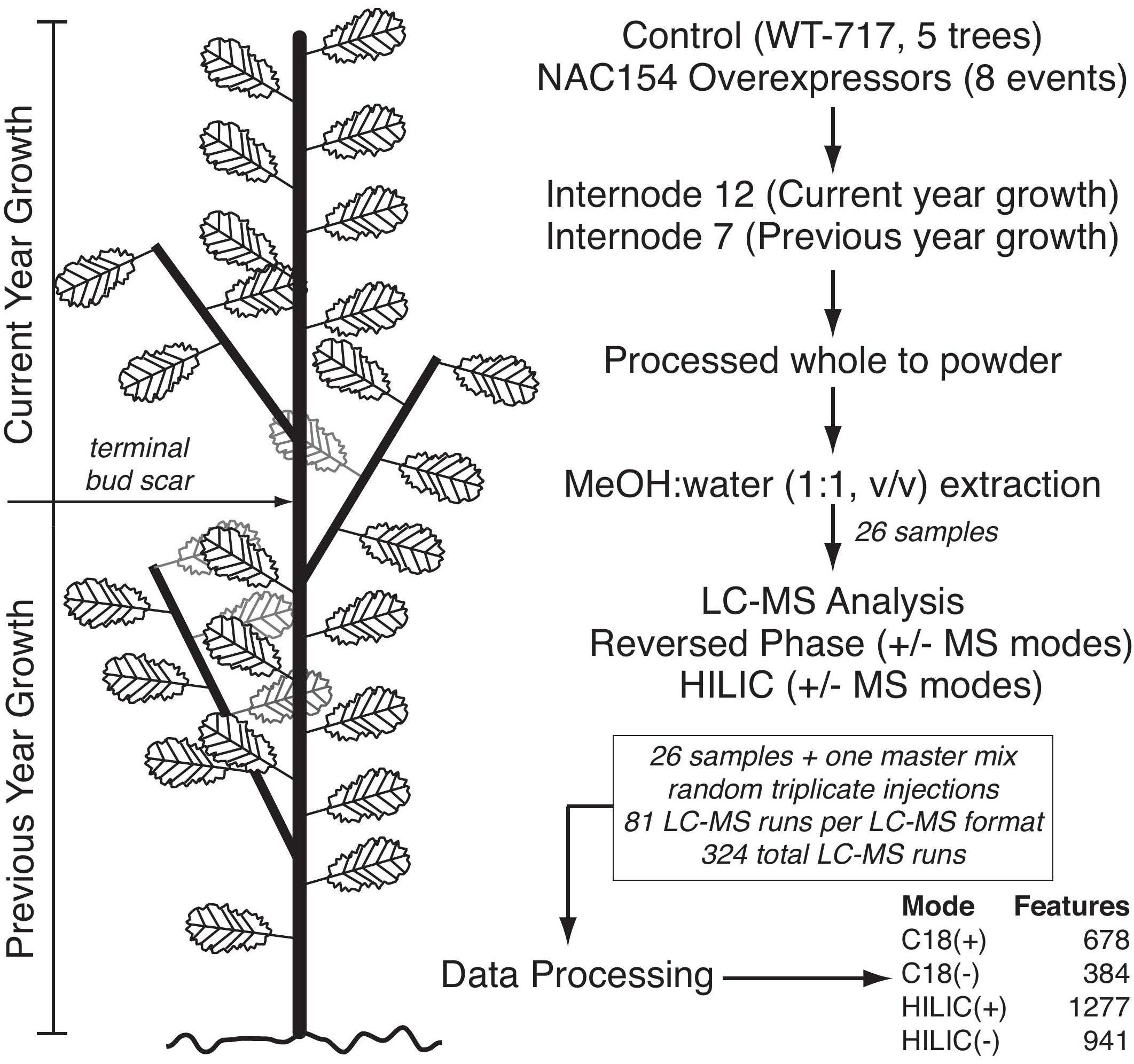




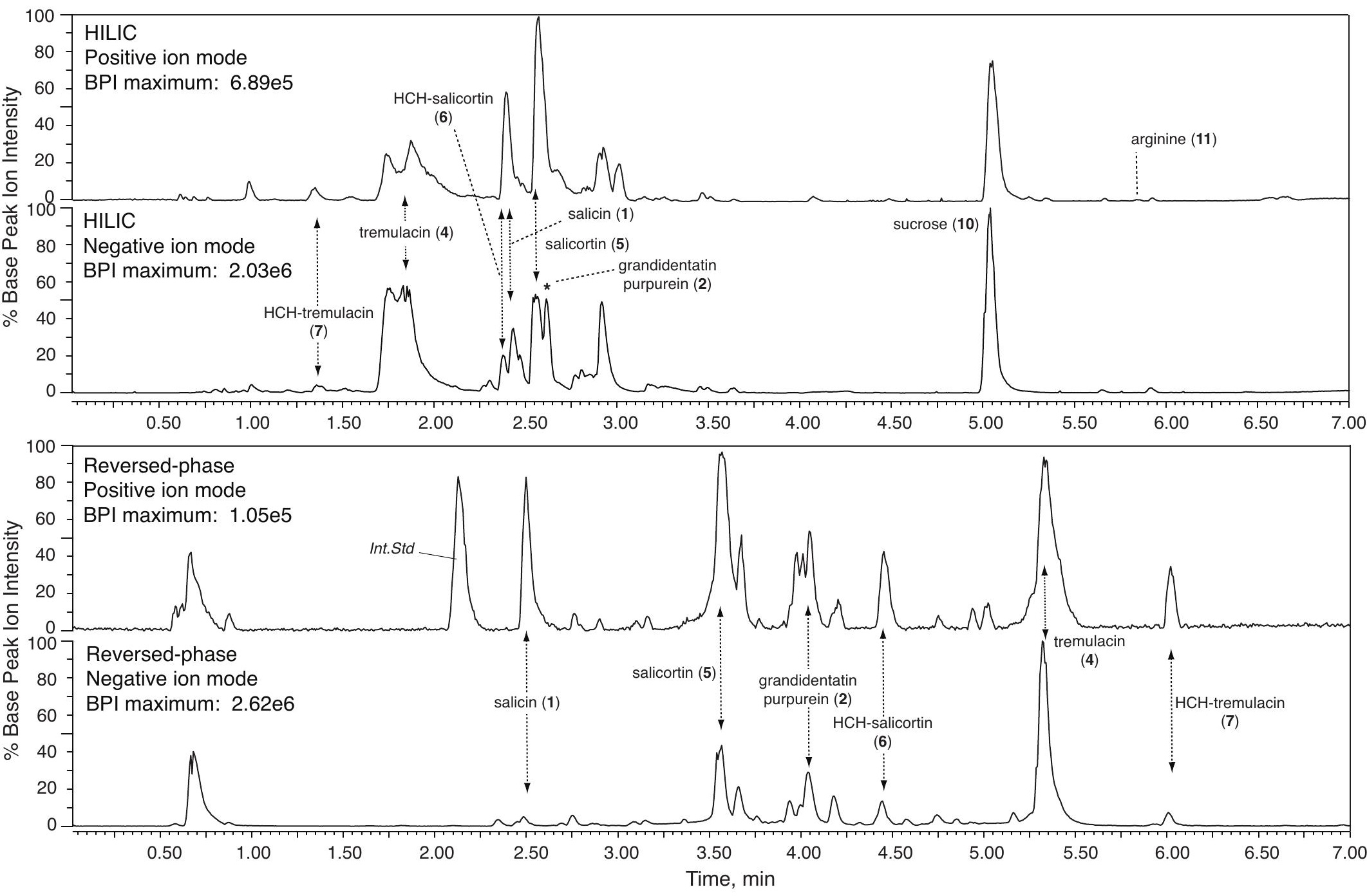



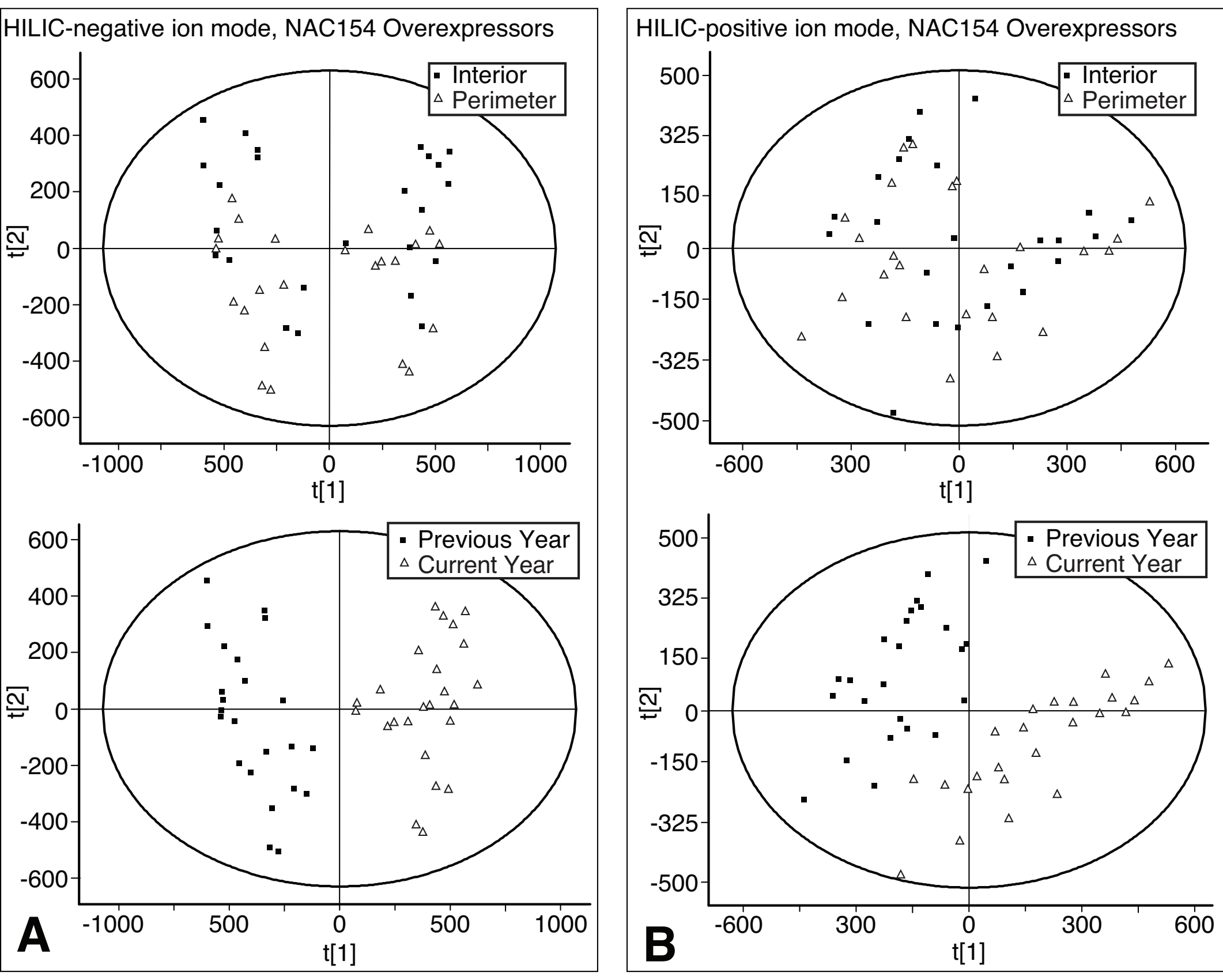

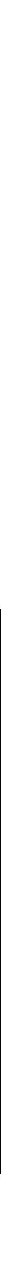